\title{
APPLICATION OF BALANCED ACCEPTANCE SAMPLING TO AN INTERTIDAL SURVEY
}

\author{
NAEIMEH ABI ${ }^{1}$, MOHAMMAD MORADI $^{2}$, MOHAMMAD SALEHI $^{3}$, JENNIFER BROWN $^{4}$, \\ JASSIM A. AL-KHAYAT ${ }^{5}$, ELENA MOLTCHANOVA ${ }^{6}$ \\ ${ }^{I}$ Dept. of Mathematics and Statistics, University of Canterbury, New Zealand. \\ ${ }^{2}$ Dept. of Statistics, College of Science, Razi University, Iran. \\ ${ }^{3}$ Dept. of Mathematics, Statistics and Physics, Qatar University, Qatar. \\ ${ }^{4}$ Dept. of Mathematics and Statistics, University of Canterbury, New Zealand. \\ ${ }^{5}$ Dept. of Biological \& Environmental Sciences, College of Arts and Science, \\ Qatar University, Qatar. \\ ${ }^{6}$ Dept. of Mathematics and Statistics, University of Canterbury, New Zealand.
}

Received: $28^{\text {th }}$ March 2017, Accepted: $4^{\text {th }}$ June 2017

\begin{abstract}
In ecological studies, the population of interest is often spread over a large area. In these studies, obtaining a sample with good spatial coverage is an important feature in the design of a survey. In most cases adjacent, or neighbouring, units are more similar than units further apart and the resulting spatial autocorrelation should be taken into account.

Two dimensional systematic sampling (grid-based sampling) is one conventional method that has been used in environmental studies to achieve spatial coverage of the area.

Balanced Acceptance Sampling (BAS) is a new method for selecting well spread out sampling units over the study area.

In this paper we will compare the BAS design and two dimensional systematic sampling for selecting samples (quadrats) from a large area, using a case study of a crab species from an intertidal marine zone in Qatar.
\end{abstract}

Keywords: Two dimensional systematic sampling, Balanced acceptance sampling, Quadrat, Crustacea, Spatial autocorrelation.

\section{INTRODUCTION}

In ecological studies, the population of interest can occupy a very large area. Therefore, obtaining a sample with good spatial coverage is one important aspect to consider in designing a survey. When adjacent or neighbouring units are more similar than units further apart, there are advantages in having a sample that is well spread out. By taking into account the presence of spatial autocorrelation the result should be a representative samples.

There are a number of different sampling strategies that can be used to help in achieving a well spread out sample (Wang et al., 2012). One of the most common methods for sampling in two dimensions is systematic sampling. Systematic sampling is used to ensure that the target population is fully and uniformly represented by the samples collected (Green, 1979). 
Although implementing a systematic sampling design is relatively easy, the existence of any unknown periodic pattern in the population may cause some issues if the interval between sampling units has the same period (Fattorini et al., 2006; Eberhardt \& Thomas, 1991). This problem is often cited as the biggest disadvantage of systematic sampling.

During the last decades, a number of sampling methods have been introduced to select samples that are spread out in a spatial context defined by geographic boundaries, what we refer to here as "well spread out" samples. Generalized Random Tessellation Stratified (GRTS) (Stevens Jr \& Olsen, 2004), Local Pivotal Methods (LPM1 and LPM2) (Grafström et al., 2012), Spatially Correlated Poisson Sampling (SCPS) (Grafström, 2012) and Balanced Acceptance Sampling (BAS) (Robertson et al., 2013) are some of these spatially balanced sampling methods.

The newest of those, BAS, is one of the simplest computational methods and, although we do not use this feature in this study, it has the added advantage that it can accommodate more than two dimensions. BAS is relatively new, and there are limited studies available in the literature where BAS has been used. L. McDonald et al. (2015) and Keinath \& Abernethy (2016) used BAS to select grid cells in different regions of the United States in order to ensure the spatial representativeness of the sample in a study of Black-Tailed prairie dogs. In another study, Howlin \& Mitchell (2016) used BAS to select locations in Bighorn Canyon National Recreation Area in order to monitor bat populations in the area. Here, we demonstrate the application of BAS to a case study of crustaceans and compare the results of the implementation with two dimensional systematic sampling method.

The article is organized as follows. A brief overview of the two dimensional systematic sampling is given in section 2. In section 3 the BAS method is introduced. The crab data set is described in section 4. In section 5 the results of applying BAS method to the crab data are reported, practical aspects are discussed in section 6 and, finally in section 7 some conclusions are drawn.

\section{TWO DIMENSIONAL SYSTEMATIC SAMPLING}

Two dimensional systematic sampling has been widely used in environmental studies. Two dimensional systematic sampling selects the initial sampling unit randomly and uses it as the origin for a regular pattern, over which the rest of the sampling units are located. Payandeh (1970) and Tomppo \& Heikkinen (1999) have used two dimensional systematic sampling in forest surveys and found that the relative efficiency of this method depends on the spatial distribution of the units. For instance, Payandeh (1970) mentioned that the systematic sampling is more precise than other sampling methods such as stratified or simple random sampling when applied to original forest areas, while systematic sampling had the least precision when applied to uniformly spaced populations such as planted production forests. Mason (1992) found that two dimensional systematic sampling in soil sampling provides a uniform coverage of the study area.

Two dimensional systematic sampling is as follows: let $A$ be a continuous region, partitioned into $R$ rows and $C$ columns such that $N(N=R \times C)$ non-overlapping rectangle sites of identical size are formed. In order to select a two dimensional systematic sample of size $n$, the $N$ quadrats have to be further stratified into $n=n_{R} \times n_{C}$ sub-regions each containing $k=k_{R} \times k_{C}$ quadrats (D’Orazio, 2003). In the simplest form of two dimensional systematic sampling, the first sampling unit (first quadrat) is selected at random from the first sub-region and the others are selected from the remaining sub-regions in the same relative position as the position of the first quadrat. 
Figure 1 illustrates an example of a two dimensional systematic sample. In this example the goal is to select a sample of size 9 . The whole population must be firstly partitioned into $9(=3 \times 3)$ equal sub-regions. One quadrat from each sub-region is selected. In this example, each sub-region contains $12(=4 \times 3)$ quadrats. The first quadrat is randomly selected from these 12 quadrats in the first sub-region and the other quadrats are chosen from the remaining sub-regions in the same position as the position of the first selected quadrat. The first sub-region and the position of the first randomly selected quadrat are shown in Figure 1 in grey colour and by a circle, respectively.

Fig. 1: An example of a two dimensional systematic sample. The first quadrat, o, is selected at random from the first sub-region. The first sub-region is shown in grey. The remaining 8 quadrats are selected from the same relative position from the 8 sub-regions

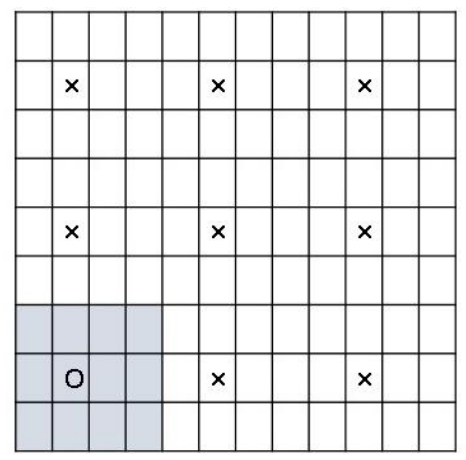

\section{BALANCED ACCEPTANCE SAMPLING}

Balanced acceptance sampling is a new spatially balanced sampling method which selects samples that are near-evenly distributed (well spread out) over the study area. This method is based on a special type of quasi-random number sequence, called the Halton sequence (Halton, 1960). The Halton sequence is constructed by partitioning the unit interval, $(0,1)$, with respect to a base $1 / p, 1 / p^{2}, 1 / p^{3}$ and so on, where $p$ is a prime number. The $d$-dimensional Halton sequence is created by a set of $d$ Halton sequences with pairwise co-prime bases (Robertson et al., 2013). As an example, the first 10 decimal members of the 2-dimensional Halton sequences are given by the sets

$$
\begin{aligned}
\{ & \left.\left(\phi_{p 1=2}(k), \phi_{p 2=3}(k)\right)\right\}_{k=1}^{10} \\
= & \{(0.5000,0.3330)(0.2500,0.6670)(0.7500,0.1110)(0.1250,0.4440)(0.6250,0.7780) \\
& (0.3750,0.2220)(.8750,0.5560)(0.0625,0.8890)(0.5625,0.0370)(0.3125,0.3700)\}
\end{aligned}
$$

In the first step of implementing the BAS method, it is necessary to specify a hyper box that encloses the study area (in this paper the study area is restricted in two dimensional space, so considering just a box is enough). Then, the set of Halton sequences according to the dimensions of interest would be calculated.

The Halton sequence is deterministic and to introduce some stochasticity in the BAS method, Robertson et al. (2013) used a random-start Halton sequence. A random-start Halton 
sequence is created by skipping some terms in the Halton sequence. For example, the random-start Halton sequence in two dimensions is achieved after skipping $u_{1}$ terms in the first dimension of the Halton sequence and $u_{2}$ terms in the second dimension of the Halton sequence, where $u_{1}$ and $u_{2}$ are two random integers generated from a uniform distribution on $[0, U]$, where $U$ is any sufficiently large integer.

The pairs, after skipping some terms, $\left(\phi_{p 1=2}\left(k+u_{1}\right), \phi_{p 2=3}\left(k+u_{2}\right)\right)$ for $k=1,2, \ldots$, can be viewed as the spatial coordinates (representing geographic locations) of a point. We call these pairs Halton points, and they are used to select the BAS sample. If the Halton point falls in a unit from the sample population the unit is selected. If not, the Halton point will be discarded and another Halton point is selected, and so on.

The BAS method was first conceived for environmental studies and surveys of natural resources. In environmental studies, often units in the study area are defined by their geographical coordinates, and for BAS each geographical axis is considered as a dimension.

Implementing BAS for selecting an equal probability sample of size $n$ in a two dimensional environmental population could be summarized as follows:

1- Specify a box that encloses the study area.

2- Generate a list of numbers from the Halton sequence in two dimensions.

3- With random starts for the two dimensional Halton sequence, create a sequence of pairs (Halton points).

4- Take each Halton point in order, and if the observed point falls in a population unit select the unit for the sample. If not, discard the Halton point and take the next point. Continue until the desired $n$ sampling units have been selected.

The algorithm to create a BAS sample is surprisingly straightforward and is available within R (R Core Team, 2016), in the package SDraw (T. McDonald, 2016).

\section{CASe STUdy}

We used a data set from a recent study of crabs from Alkhor, in the intertidal zone on the east coast of Qatar. In a field study in March 2014, a sample of 80 quadrats was selected. The number of open crab-burrows of the species Nasima dotilliformis was counted in each $1 \mathrm{~m}^{2}$ quadrat.

The sample was selected by placing 12 parallel strips at equal distances apart and taking a systematic sample from each strip, with 6 or 7 quadrats per strip. The latitude and longitude were recorded for each selected quadrat or sample unit.

We used this information on the quadrat counts and locations to create a synthetic population of crabs using a Nadaraya-Watson smoother with Gaussian kernel weighting (Nadaraya, 1964; Watson, 1964 and Nadaraya, 2012). This function can be found in the package spatstat in R (R Core Team, 2016). The generated counts of Nasima dotilliformis were a $400 \times 400$ matrix, representing the survey population for the study area, which each entry in the matrix considered the count of crabs in a quadrat. Here we assume each crab-burrow represents one crab, so that the count of burrows is synonymous with the count of crabs in our synthetic population. 
To compare designs we sampled the population by selecting quadrats with two different methods: two dimensional systematic sampling and BAS. Simple random sampling (SRS) method was also considered as a reference method for comparing the results.

To carry out the two dimensional systematic sampling, the population was partitioned into equal sub-regions according to the desired sample size. For example in the case with a sample size of $n=36$, the longitude was partitioned into 6 equal intervals, and 36 equal sub-region would be created. The first quadrat was randomly selected from the first sub-region. Other quadrats in the remaining sub-regions had the same position as the first sampling unit within the sub-region.

\section{Spatial coverage}

Voronoi polygons can be used as a method for measuring how well spread out a sample is. This method is based on the distance between elements selected in the sample $(s)$. The voronoi polygon $p_{\mathrm{i}}$, for $i \in s$, includes all population units $j$ satisfying $d\left(x_{i}, x_{j}\right)<d\left(x_{k}, x_{j}\right)$ for all sampling units $k \in s$, where $d\left(x_{i}, x_{j}\right)$ indicates the distance between units $\mathrm{i}$ and $\mathrm{j}$. If $d\left(x_{i}, x_{j}\right)=d\left(x_{k}, x_{j}\right)$, then unit $j$ is included in both $p_{i}$ and $p_{k}$. As a simple example, consider a population with three units (labeled $A, B$ and $C$ ). The units $A$ and $B$ are selected in the sample, and unit $C$ is not. Let $D_{1}$ be the distance between $A$ and $C$ and $D_{2}$ be the distance between $B$ and $C$. If $D_{1}<D_{2}$ then $C \in p_{A}$, If $D_{2}<D_{1}$ then $C \in p_{B}$ and if $D_{2}=D_{1}$ then $C \in p_{B}$ and $C \in \mathrm{p}_{\mathrm{A}}$, simultaneously. Three different styles of voronoi polygon which could be created in this example are shown in the Figure 2.

Fig. 2: Three different Voronoi polygons which could be created in a population by three units. Unit $A$ and $B$ are selected in the sample. (a) $D_{1}<D_{2}$ (b) $D_{1}>D_{2} \quad$ (c) $D_{1}=D_{2}$

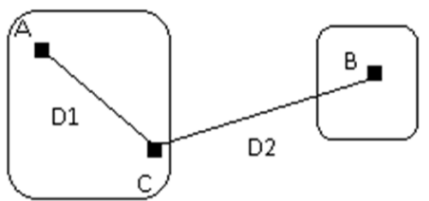

a) $\mathrm{D} 1<\mathrm{D} 2$

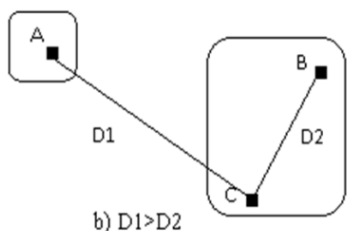

b) $\mathrm{D} 1>\mathrm{D} 2$

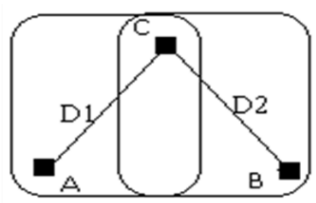

c) $\mathrm{D} 1=\mathrm{D} 2$

Let $n_{i}$ and $v_{i}$ denote the number of units in $p_{i}$ and the sum of inclusion probability in $p_{i}$, respectively. If unit $j$ is included in $m_{j}$ polygon, then $j$ is counted as $\frac{1}{m_{j}}$ and its inclusion probability is divided equally to each of the $\mathrm{m}_{\mathrm{j}}$ polygons.

After deriving the voronoi polygons for a given sample, the variance of the voronoi polygon is calculated by:

$$
V(v)=\frac{1}{n} \sum_{\mathrm{j} \in \mathrm{s}}\left(v_{\mathrm{j}}-1\right)^{2},
$$

where $v_{j}$ indicates the sum of the inclusion probabilities of units in the Voronoi polygon related to the $j^{\text {th }}$ sampling unit. Stevens Jr \& Olsen (2004) used this measure as an indicator 
of spatial spread for a given sample where small values of $\mathrm{V}(v)$ indicate a very well spread sample.

In our study, for each sample size, the process of selecting samples was repeated 1000 times, and $V(v)$ calculated for each. To compare $V(v)$ among different sampling schemes, we calculated the average of the $V(v)$ for the 1000 replications:

$$
\hat{\mu}(\mathrm{V}(v))=\frac{1}{1000} \sum_{\mathrm{r}=1}^{1000} \mathrm{~V}\left(\mathrm{v}_{\mathrm{r}}\right),
$$

where $\mathrm{V}\left(\mathrm{v}_{\mathrm{r}}\right)$ is the Voronoi polygon of the $r^{\text {th }}$ iteration. Small $\hat{\mu}(\mathrm{V}(v))$ indicates a well spread out sample, and one with spatial balance.

\section{Parameter estimation}

Estimation of population characteristic(s) is usually the final aim of the sampling surveys. In this study, the total number of crabs in the study area is the parameter of interest. The Horvitz-Thompson (HT) estimator (Horvitz \& Thompson, 1952) can be used for estimating the total number of crabs in the study area as follow:

$$
\widehat{\mathrm{Y}}_{\mathrm{HT}_{-} \mathrm{i}}=\sum_{\mathrm{j}=1}^{\mathrm{n}} \frac{\mathrm{y}_{\mathrm{j}}}{\pi_{\mathrm{j}}},
$$

where $\widehat{Y}_{\mathrm{HT}_{-} \mathrm{i}}$ is the total number of crabs estimated from the $\mathrm{i}^{\text {th }}$ iteration. Also, $\mathrm{y}_{\mathbf{j}}$ and $\pi_{\mathrm{j}}$ are the number of crabs and the inclusion probability related to the $\mathrm{j}^{\text {th }}$ sample quadrat, respectively.

In this sub-section, we compare the variance of the HT estimator achieved from 1000 simulated samples in three different sampling schemes. The variance of the HT estimator for 1000 simulated sample are estimated by:

$$
\widehat{\operatorname{Var}}\left(\widehat{Y}_{H T}\right)=\frac{1}{1000} \sum_{\mathrm{i}=1}^{1000}\left(\widehat{Y}_{\mathrm{HT}_{-} \mathrm{i}}-\mathrm{Y}\right)^{2} .
$$

Since this study is based on simulation, Y therefore represents the true total number of crabs in the study area, 1336781.

We used $\widehat{\operatorname{Var}}\left(\widehat{Y}_{H T}\right)$ to show how precise a sampling method is, with smaller values for more precise sampling method.

\section{RESULTS}

The $\hat{\mu}(V(v))$ for three different sampling schemes are shown in first column of Table 1 . The smallest and largest value of $\hat{\mu}(\mathrm{V}(v))$ is related to the two dimensional systematic sampling and simple random sampling method, respectively. The very low value of $\hat{\mu}(V(v))$ for systematic sampling can be explained by the survey patterns. For example with a sample of size $n=48$, the quadrats (sampling units) which are selected by two dimensional systematic sampling method have a regular pattern in comparison with two other methods (Figure $3 b$ ). This pattern is obvious in Figure 3b. Figure 3a shows how a sample from BAS and it is more evenly spread than the sample from SRS (Figure 3c). 
Fig. 3: A sample of size equal to 48 quadrats according to (a) BAS method, (b) two dimensional systematic sampling method and (c) simple random sampling method, respectively

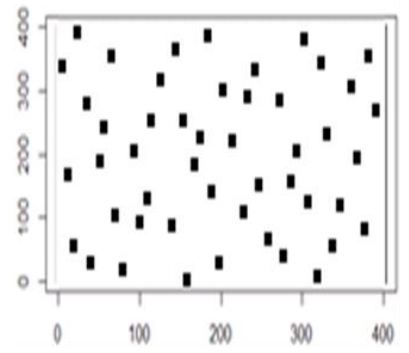

(a)

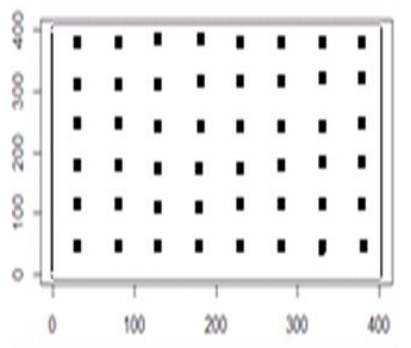

(b)

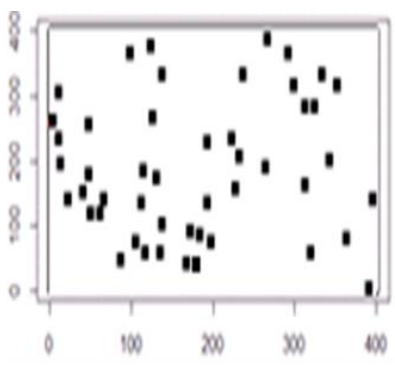

(c)

The $\widehat{\operatorname{Var}}\left(\widehat{Y}_{H T}\right)$ for the three different sampling schemes, with the different sample sizes are shown in the last column of Table 1.

Table 1 The average of $V(v)$ and the variance of the HT estimator for three sampling schemes with different sample sizes. The first column is the average of the $V(v)$, the sampling method by the smaller values of $\widehat{\mu}(V(v))$ is considered to be more spatially balanced. The second column contains the variance of the HT estimator. The method by the smallest value of variance is more precise

\begin{tabular}{|ccrr|}
\hline $\mathrm{n}$ & design & \multicolumn{1}{c}{$\hat{\mu}(V(v))$} & $\widehat{\operatorname{Var}}\left(\widehat{Y}_{H T}\right)$ \\
\hline \multirow{3}{*}{36} & BAS & $6000 \times 10^{-5}$ & $114 \times 10^{8}$ \\
& SRS & $30000 \times 10^{-5}$ & $406 \times 10^{8}$ \\
& SYS & $5 \times 10^{-5}$ & $85 \times 10^{8}$ \\
\hline \multirow{3}{*}{81} & BAS & $6000 \times 10^{-5}$ & $39 \times 10^{8}$ \\
& SRS & $30000 \times 10^{-5}$ & $185 \times 10^{8}$ \\
& SYS & $5 \times 10^{-5}$ & $38 \times 10^{8}$ \\
\hline \multirow{3}{*}{121} & BAS & $6000 \times 10^{-5}$ & $12 \times 10^{8}$ \\
& SRS & $30000 \times 10^{-5}$ & $113 \times 10^{8}$ \\
& SYS & $10 \times 10^{-5}$ & $27 \times 10^{8}$ \\
\hline \multirow{3}{*}{169} & BAS & $6000 \times 10^{-5}$ & $6 \times 10^{8}$ \\
& SRS & $30000 \times 10^{-5}$ & $81 \times 10^{8}$ \\
& SYS & $1 \times 10^{-5}$ & $17 \times 10^{8}$ \\
\hline \multirow{3}{*}{196} & BAS & $7000 \times 10^{-5}$ & $6 \times 10^{8}$ \\
& SRS & $30000 \times 10^{-5}$ & $77 \times 10^{8}$ \\
& SYS & $2 \times 10^{-5}$ & $16 \times 10^{8}$ \\
\hline \multirow{2}{*}{256} & BAS & $7000 \times 10^{-5}$ & $3 \times 10^{8}$ \\
& SRS & $30000 \times 10^{-5}$ & $65 \times 10^{8}$ \\
& SYS & $10 \times 10^{-5}$ & $12 \times 10^{8}$ \\
\hline \multirow{2}{*}{289} & BAS & $6000 \times 10^{-5}$ & $1 \times 10^{8}$ \\
& SRS & $30000 \times 10^{-5}$ & $46 \times 10^{8}$ \\
& SYS & $1 \times 10^{-5}$ & $10 \times 10^{8}$ \\
\hline
\end{tabular}


Fig. 4: The $\widehat{\operatorname{Var}}\left(\widehat{Y}_{H T}\right)$ achieved by three different sampling methods with different sample sizes

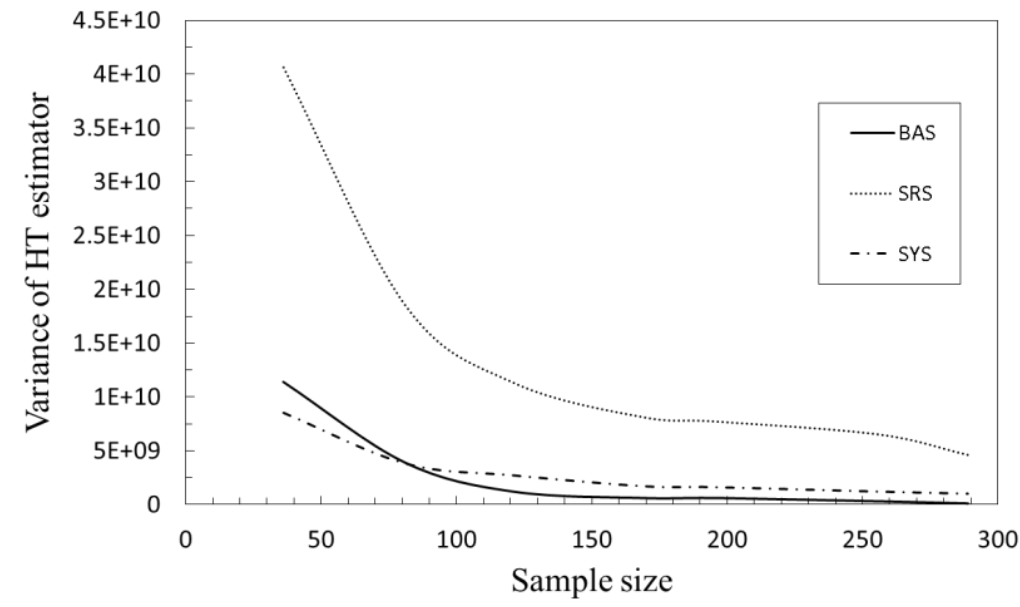

The trends of the $\widehat{\operatorname{Var}}\left(\widehat{Y}_{H T}\right)$ for the different sampling methods and sample sizes are shown in Figure 4. With increasing sample size, the variance of all of these methods decreased, and both BAS and two dimensional systematic sampling had lower variance than simple random sampling. These two designs had similar estimated variance, with BAS being more precise of the two dimensional sampling, except with the smaller sample sizes.

\section{FURTHER DISCUSSIONS ABOUT BAS}

Of the three designs we used in our study, BAS and two dimensional systematic sampling were superior to simple random sampling in terms of spatial spread and precision. In addition to these statistical advantages, there are a number of practical considerations. Encountering unforeseen factors is a common issue in implementing sampling methods on environmental fields; therefore designing a flexible method which could adapt to field changes is desirable.

Generally in two dimensional systematic sampling method, the quadrats are selected with a fixed distance between quadrats and with a regular pattern. Full coverage of the study area will only be met once the sampling process is completed. In some field situations, completing the entire sampling process may not be possible, for example if bad weather stops the field surveys early. In this situation, two dimensional systematic sampling may not have consistent spread of the quadrats over the study area and there may be gaps where quadrats are not visited. BAS, on the other hand, is able to cover the study area even when sampling is stopped early if quadrats are visited in the order they were generated. For more clarity, assume that because of an extraordinary event we are forced to stop the sampling process at 30 quadrats instead of 48 . Fig. 5 shows the quadrats that will be visited by a) BAS and b) two dimensional systematic sampling if the site ordering is strictly followed. 
Fig. 5: The resultant survey when only 30 quadrats instead of 48 quadrats are selected with (a) BAS and (b) two dimensional systematic sampling method, respectively

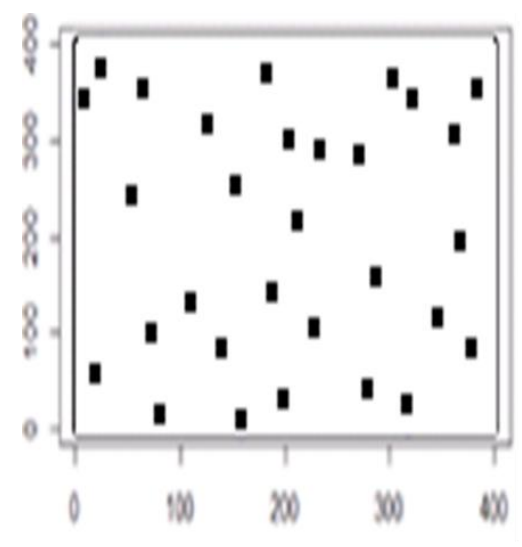

(a)

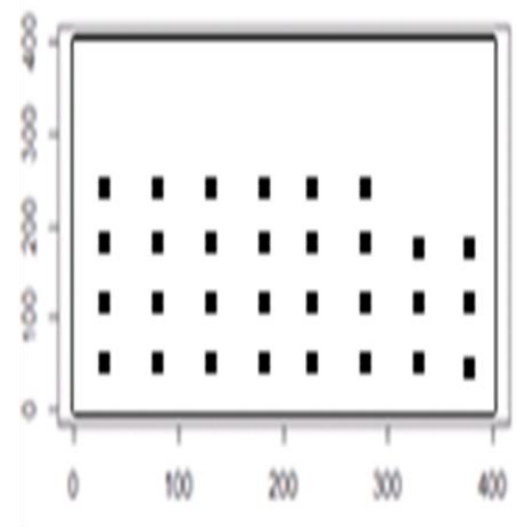

(b)

There is still an acceptable coverage of study area by the BAS method if the quadrats are visited in the sequential order, whereas two dimensional systematic sampling method is not able to provide coverage over the study area while the sampling process has been stopped.

In practice, visiting BAS quadrats in their exact order would be very time consuming and costly because the study area would be traversed many times. Instead we recommend sets of quadrats are visited. For example, a set could be 5 quadrats (quadrats 1-6, quadrats 7-12 and so on), where the time taken to survey a set relates to a practical unit in time (a half-day for example). In this way, as long as a set is complete there will always be site coverage. To illustrate this, in our study, the quadrats could be partitioned into 8 sets and all sites are visited within each set following the most practical travel path.

Other practical advantages of BAS method over two dimensional systematic sampling method are as follows,

i. In environmental samples, access to some sampling units maybe denied or is impossible. These sampling units are considered as missing values. With BAS, new sampling units can be easily substituted for the missing sampling units. The new sampling unit is added to the sample by continuing the sampling selection process. With two dimensional systematic sampling method adding new sampling units to substitute inaccessible sampling units may lead to a loss of spatial balance, especially if there are a considerable number of inaccessible sampling units.

ii. In situations where there is a change to the sample size during the survey, e.g. extra resources are allocated to the study, spatial spread can be achieved with BAS by continuing the sampling selection process. In contrast, with the two dimensional sampling method is more difficult to add extra sampling units without disrupting the regular pattern (Stehman, 2009), unless the count of the extra units is a multiple of the sample size. In fact any sample size can be accommodated in BAS, whereas a sample size that is a prime number is not easily possible in two dimensional systematic sampling method.

iii. In implementing the two dimensional systematic sampling method, the study area is partitioned into sub-regions and then sampling units are selected from each of them. When the study area does not have a regular shape, some sampling units may be missed. Figure 6 shows the sampling units which are selected by two dimensional systematic 
sampling method from a study area with irregular shape (the study area is shown by grey colour). The area within which the study area sits is divided into 9 sub-regions. In Figure $6 \mathrm{a}$, the resultant sample size is only $n=5$ because many sampling units are outside the study area. With such a predetermined pattern for selecting sampling units the actual sample size is variable. Consider when the grid is overlaid in a different orientation and different units are selected in the sub-regions. The resultant sample size in Figure $6 \mathrm{~b}$ is now $n=3$.

Fig. 6: Examples of sampling units achieved by two dimensional systematic sampling method from a study area with irregular shape (a) the resultant sample size $=5$ (b) the resultant sample size $=3$

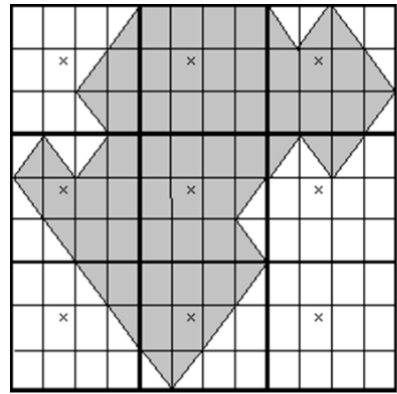

(a)

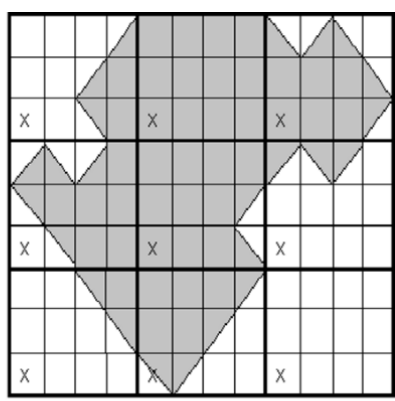

(b)

BAS on the other hand, uses the acceptance/rejection sampling technique and discards the selected sampling units which are located outside the study area and the sample size is predetermined and fixed.

iv. Other benefits of BAS are that sampling units can be selected by unequal selection probabilities. In survey sampling, using auxiliary variables to determine unequal selection probabilities for sample units can be very helpful, for example, to increase sample effort in favourable habitats and to decrease it in unfavourable habitats.

\section{CONCLUSION}

BAS is a new method that can be used for selecting sample that is well spread out over the population. In this paper, BAS has been used for selecting quadrats to estimate the size of a crab population, and the results have been compared with two dimensional systematic sampling method. The two methods were comparable, but given the practical advantages of BAS, we would recommend the use of the new design. To achieve a well spread out sample by two dimensional systematic sampling method usually every sample unit must be visited With BAS the sampling process can stop at any time and the result will still be a well spread out sample (as long as units are visited in the order given by BAS, or there about). The flexible characteristics of BAS such as being able to accommodate missed sampling units and the ability to add extra sampling units during survey implementation offers many practical benefits to ecologists. Finally, balanced acceptance sampling is able to be used for any sample size whereas two dimensional systematic sampling cannot be used easily if $n$ is a prime number. 


\section{REFERENCE}

D'Orazio, M. (2003). Estimating the variance of the sample mean in two-dimensional systematic sampling. Journal of Agricultural, Biological, and Environmental Statistics, 8(3), 280-295.

Eberhardt, L., \& Thomas, J. (1991). Designing environmental field studies. Ecological Monographs, 61(1), 53-73.

Fattorini, L., Marcheselli, M., \& Pisani, C. (2006). A three-phase sampling strategy for large-scale multiresource forest inventories. Journal of Agricultural, Biological, and Environmental Statistics, 11(3), 296-316.

Grafström, A. (2012). Spatially correlated Poisson sampling. Journal of Statistical Planning and Inference, 142(1), 139-147.

Grafström, A., Lundström, N. L., \& Schelin, L. (2012). Spatially balanced sampling through the pivotal method. Biometrics, 68(2), 514-520.

Green, R. H. (1979). Sampling design and statistical methods for environmental biologists: John Wiley \& Sons.

Halton, J. H. (1960). On the efficiency of certain quasi-random sequences of points in evaluating multi-dimensional integrals. Numerische Mathematik, 2(1), 84-90.

Horvitz, D. G., \& Thompson, D. J. (1952). A generalization of sampling without replacement from a finite universe. Journal of the American statistical Association, 47(260), 663-685.

Howlin, S., \& Mitchell, J. (2016). Monitoring Black-Tailed Prairie Dogs in Colorado with the 2015 NAIP Imagery.

Keinath, D. A., \& Abernethy, I. (2016). Bat population monitoring of bighorn canyon national recreation area: 2015 progress report.

Mason, B. J. (1992). Preparation of soil sampling protocols: sampling techniques and strategies. Retrieved from

McDonald, L., Mitchell, J., Howlin, S., \& Goodman, C. (2015). Range-Wide Monitoring of Black-Tailed Prairie Dogs in the United States: Pilot Study.

McDonald, T. (2016). SDraw: Spatially Balanced Sample Draws for Spatial Objects. R package version 2.1.3. https://CRAN.R-project.org/package=SDraw.

Nadaraya, E. (1964). On estimating regression. Theory of Probability \& Its Applications, 9(1), 141-142.

Nadaraya, E. (2012). Nonparametric estimation of probability densities and regression curves (Vol. 20): Springer Science \& Business Media.

Payandeh, B. (1970). Relative efficiency of two-dimensional systematic sampling. Forest science, 16(3), 271-276.

R Core Team, (2016, September). A language and environment for statistical computing. R Foundation for Statistical Computing, Vienna, Austria. 2015. Retrieved September 5, 2016, from URL http. www.R-project.org.

Robertson, B., Brown, J., McDonald, T., \& Jaksons, P. (2013). BAS: Balanced acceptance sampling of natural resources. Biometrics, 69(3), 776-784.

Stehman, S. V. (2009). Sampling designs for accuracy assessment of land cover. International Journal of Remote Sensing, 30(20), 5243-5272.

Stevens Jr, D. L., \& Olsen, A. R. (2004). Spatially balanced sampling of natural resources. Journal of the American statistical Association, 99(465), 262-278. 
Abi N., Moradi M., Salehi M., Brown J., Al-Khayat J.A., Moltchanova E.: Application of Balanced Acceptance Sampling to an Intertidal Survey

Tomppo, E., \& Heikkinen, J. (1999). National forest inventory of Finland — past, present and future. Statistics, registries and research-experiences from Finland, 89-108.

Wang, J.-F., Stein, A., Gao, B.-B., \& Ge, Y. (2012). A review of spatial sampling. Spatial Statistics, 2, 1-14.

Watson, G. S. (1964). Smooth regression analysis. Sankhyā: The Indian Journal of Statistics, Series A, 359-372. 